\title{
Moisture, a Vital but Neglected Factor in the Seasonal Ecology of Insects: Hypotheses and Tests of Mechanisms
}

\author{
MAURICE J. TAUBER, ${ }^{1}$ CATHERINE A. TAUBER, ${ }^{1}$ JAN P. NYROP, ${ }^{2}$ aND MICHAEL G. VILLANI ${ }^{2}$
}

\begin{abstract}
Environ. Entomol. 27(3): 523-530 (1998)
ABSTRACT Of the major physical factors that influence insect seasonal ecology, moisture is least understood and least appreciated. It is our premise that experimental probing of insects from diverse zones and various habitats would reveal general patterns of insect responses to moisture that are as striking as those for photoperiod and temperature. Using the paradigms of photoperiod and temperature as ecophysiological determinants of insect seasonality, we hypothesize that moisture influences insect life cycles via one or more of three mechanisms-as a token stimulus for diapause, modulator of developmental or reproductive rates, or behavioral cue for vital seasonal events. For heuristic purposes, we offer each of these hypotheses in close association with approaches for testing their validity in insects that undergo dry-season dormancy. The approaches appear appropriate for examining the role of moisture in the life histories of terrestrial invertebrates other than insects, as well as plants and microbes that have a seasonal resting stage. Elucidating moisture's role in insect seasonal cycles is critical to the development of comprehensive phenological models, improved insect management systems, and identification of novel evolutionary mechanisms for adaptation to wet-dry seasons, especially in tropical, subtropical, and Mediterranean regions.
\end{abstract}

KEY WORDS phenology, moisture, soil-inhabiting insects, dormancy, development, reproduction

PhOTOPERIOD AND TEMPERATURE are generally considered prime factors in the seasonal ecology of terrestrial invertebrates (Beck 1980, Tauber et al. 1986). In contrast, moisture is usually consigned a secondary or minor role. This subordinate ranking of moisture as a seasonal cue may be grossly misleading for 2 reasons: First, phenological studies are concentrated in the Temperate Zone where large seasonal changes in photoperiod and temperature typically occur. Consequently, investigators are heavily biased toward using photoperiod and temperature as variables, and data on moisture's function and significance are lacking. Second, the natural histories of a large proportion of earth's insects (e.g., those from tropical, Mediterranean, and other regions with conspicuous wet and dry seasons) are highly correlated with changes in moisture conditions (Essig 1926, Edney 1977, Wolda 1978, Shapiro 1979, Mooney et al. 1980, Labeyrie 1981, King 1984, Denlinger 1986, Paarmann 1986, Powell 1986, Brakefield 1987, Dobkin et al. 1987, Janzen 1987, Bradshaw and Holzapfel 1988, Cloudsley-Thompson 1991, Hadley 1994, Sømme 1995). Indeed, it appears that cycles of rainfall and moisture in wet-dry regions may rival those of temperature in temperate regions, in both their seasonal predictability and their importance for survival and development of insects. Thus, we contend that moisture constitutes a greatly un-

\footnotetext{
${ }^{1}$ Department of Entomology, Comstock Hall, Cornell University, Ithaca, NY 14853

2 Department of Entomology, Barton Laboratory, NYSAES, Cornell University, Geneva, NY 14456.
}

derappreciated and poorly understood proximate and ultimate determinant of insect life-histories. And, we propose that an experimental probing of insect responses to moisture would lead to a more balanced view of how the major physical factors mold the lives of insects.

A fuller evolutionary and mechanistic understanding of moisture's function as a seasonal cue has considerable ecological and practical significance. For example, it would strengthen our ability to predict and manipulate the seasonal activity of organisms from regions with distinct wet-dry seasons; these organisms include insect pests and natural enemies, as well as insectivorous vertebrates (Spiller and Schoener 1995). Furthermore, it would enable ecologists to assess the consequences of environmental perturbation (e.g., global warming, alterations in annual rainfall patterns, and the introduction of irrigation or periodic flooding into arid regions) (Mattson and Haack 1987, Cammell and Knight 1992). And finally, if conducted within a phylogenetic setting, comparative studies that focus on the mechanisms of response to moisture could help elucidate evolutionary pathways of adaptation to long-term climatic changes.

Until recently, the tantalizing notion of defining patterns of response to moisture has been elusive because of formidable technical and conceptual difficulties. Here, we propose a practical experimental framework for assessing the impact of seasonal changes in moisture on insect life cycles. Our intent is to stimulate experimentation with moisture as a seasonal cue, especially, but not exclusively, in tropical 
Table 1. Seasonal responses to moisture: hypotheses and mechanisms

Our 3 hypotheses concerning moisture's role as a seasonal factor cover the entire life cycle, and they are broadly applicable to a wide variety of organisms that encounter seasonal changes in moisture.

1. Token stimulus hypothesis: Moisture acts as a token

(anticipatory) stimulus that induces, maintains or terminates diapause.

Moisture's effects on diapause can be measured through multiple responses-through its influence on the expression of dormancy, movement, or other diapause-associated behavior, cold-, heat-, or drought-hardiness, as well as morphological structure or color (seasonal polyphenism) (e.g., see Tauber et al. 1986, Danks 1987, Lee and Denlinger 1991, Brakefield and Reitsma 1991). A token stimulus is an environmental cue that in itself is not favorable or unfavorable for development or reproduction but that heralds a change in environmental conditions (e.g., an anticipatory cue that induces, maintains, or terminates diapause (e.g., Tauber et al. 1986]). Unlike photoperiod (day length), which acts primarily as a token stimulus, temperature, food, and moisture can have both direct and token effects on development or behavior. As a result, it is a significant experimental challenge to isolate or separate the mechanisms through which these factors influence seasonal cycles (Ando 1972, Tauber et al. 1986).

2. Developmental modulator hypothesis: Moisture acts to modulate development or activity; that is, it accelerates, decelerates, or limits rates of growth, maturation, oviposition, or behavior (e.g., movement, feeding).

When acting as a developmental modulator, moisture is expected to interact strongly with temperature, food, and other biotic factors.

3. Behavioral cue hypothesis: Moisture acts as a primary stimulus for crucial seasonal activities in insect life cycles (e.g., postdiapause hatching, moulting, mating, movement).

Given that moisture can influence more than one physiological or behavioral process during an insect's life cycle, the above hypotheses are not mutually exclusive.

and subtropical regions, and to provide a conceptual basis for comparative studies aimed at evolutionary analyses of insect adaptations to the full range of major physical factors. Clearly, moisture can interact with temperature, photoperiod, and food to influence insect life cycles, but our approach here is to concentrate on moisture as an independent variable. Although our emphasis is on insects, an analogous approach is appropriate for other terrestrial invertebrates, as well as plants and microbes that have seasonal resting stages (dormant seeds or spores).

Using the paradigms of photoperiod and temperature as ecophysiological determinants of insect seasonality, we hypothesize that moisture influences insect life cycles via 1 or more of 3 mechanisms (Table 1). For heuristic purposes, we offer each of these hypotheses in close conjunction with approaches for testing their validity. Within each of the 3 mechanisms, moisture may act in a quantitative manner or as an all-or-none stimulus, and it may have a primary role in determining seasonality or it may modify temperature or photoperiod (Tauber et al. 1986). Moreover, it is noteworthy that for some insects, ingestion of water (Beck 1980), including moisture within food, or absorption of water vapor from the atmosphere without any contact water (Yoder and Denlinger 1992) are important in the perception of moisture as a seasonal cue; in others, contact with free water (rainfall) pro- vides a key seasonal stimulus (Wolda and Denlinger 1984, Okuda 1990).

\section{Moisture as a Token Stimulus for Diapause}

In no case has insect responsiveness to moisture as a seasonal cue for diapause been examined to the degree that parallels responsiveness to photoperiod or temperature. The roles of photoperiod and temperature as token stimuli in inducing, maintaining, and terminating diapause have been shown for hundreds of insect species, and it is clear that striking patterns of response are shared across diverse taxa (e.g., Beck 1980, Saunders 1982, Tauber et al. 1986, Danks 1987). Insects show sensitivity to these 2 environmental stimuli during specific periods in their life cycles, and they react by entering diapause at a later time; some tropical and subtropical animals can respond to remarkably subtle changes in day length or temperature (Denlinger 1986). Moreover, in some cases, comparative studies of closely related species and geographically diversified populations have identified the genetic basis for evolutionary changes in responses (e.g., Tauber et al. 1986, Tauber and Tauber 1992).

In marked contrast, generalizations describing moisture's influence on diapause remain elusive. Water relations during diapause and water's role in coldand heat-hardiness have been studied, but the action of moisture as a token (anticipatory) cue is rarely confirmed or excluded (Masaki 1980, McLeod 1984, Denlinger 1986, Tauber et al. 1986, Janzen 1987, Jones 1987, Wolda 1989, Lee and Denlinger 1991). In some cases, the water content of food appears to provide seasonal cues (Tauber et al. 1986). However, it is generally assumed that because of spatial and temporal variability in its occurrence, moisture rarely serves as a cue for diapause induction. Not only is this assumption questionable, but it is seldom tested, even for insects from regions with regular wet and dry seasons (Wardhaugh 1980, Tanaka 1987). Moreover, it is well known that for large numbers of species, moisture-related cues stimulate emergence or the resumption of development at the end of dormancy. Despite this phenomenon, few studies have either looked for seasonal patterns of responsiveness to moisture or sought to identify the role of moisture as a token stimulus in diapause maintenance or termination (references in Denlinger 1986 and Tauber et al. 1986; Ingrisch 1986; Janzen 1987; Jones 1987; Tanaka 1987; Tanaka et al. 1987a, b; Gebre-Amlak 1989; Gehrken 1989; van Houten and Veenendaal 1990; Okuda 1990, 1991; Kfir 1993). Results from these few cases indicate that moisture can influence insect life cycles in diverse ways; they also underscore the need for comparative studies dealing with moisture and for investigations on the interaction between moisture and other seasonal cues in the expression of diapause.

\section{Moisture and Development}

In no case has moisture's influence on insects' seasonal patterns of development or activity been quan- 
tified in a manner that parallels that of temperature or photoperiod. Within defined upper and lower limits, developmental and activity rates of poikilothermic animals are usually linearly related to temperature, and within these limits, heat accumulation can help predict phenological events (e.g., Wagner et al. 1991). Most phenological models are entirely temperaturedriven, and they vary greatly in their accuracy for describing seasonal development and activity of insects. In some cases, the role of photoperiod is included (Gutierrez et al. 1986, Taylor 1989).

With notable exceptions, developmental responses to moisture remain largely untested. In cases where it has been studied, moisture by itself or by interacting with temperature, food or soil texture, was shown to influence rates of development or activity (Fletcher et al. 1978, Régnière et al. 1979, Wilson et al. 1982, Punyua et al. 1985, Jordan and Hubbard 1991, Bonato et al. 1995). Thus, considerable opportunities exist for exploring moisture's role and incorporating these responses into phenological models (Chu et al. 1992a, b).

\section{Moisture and Seasonal Behavior}

Moisture's function as a major cue for seasonal behavior is relatively unexplored. For many insects, the ending of dormancy encompasses a 2-phase process. Sometime prior to the end of the unfavorable period, diapause (or an early phase of diapause) terminates, but subsequent postdiapause development and activity are curtailed until favorable physical conditions return. Temperature's role in ending this 2 nd phase of dormancy and in promoting postdiapause activity has been well studied (Tauber et al. 1986).

In contrast, despite numerous observations that the initiation of insect activity after dormancy ends is strongly correlated with moisture conditions (see references above), moisture's function as a cue for the expression of insect seasonal behavior is poorly understood. In very few cases has moisture's influence as a stimulus for postdiapause behavior been distinguished from its function in diapause termination (e.g., Beck 1980, Tanaka et al. 1987a). These mechanisms would have markedly different life-history consequences, and yet few investigations attempt to differentiate between them (Ando 1972, Tauber et al. 1986, 1994).

\section{Evaluation of Moisture as a Seasonal Factor}

Choosing Experimental Animals. Given the diversity of insect species that experience wet-dry seasons, it is useful to ask which among them are prime candidates for examining experimentally moisture's role as a seasonal cue? We conclude that, as a group, insects that inhabit soil, wood, or other nonliving relatively solid media can serve this function particularly well for at least 2 reasons. First, most insects whose life cycles are synchronized with seasonal changes in moisture spend a considerable period of dormancy in the soil, leaf litter, or other substrate associated with soil (Janzen 1983, 1987; for insects associated with wood, see
Parkin 1943, Becker 1977, Williams 1983). These include species from diverse taxonomic groups, species with dormancy in all life stages, and species having wet-season dormancy, as well as the more common dry-season dormancy. Thus, we predict that comparative studies of species found in relatively solid media would unearth a variety of response patterns and underlying mechanisms.

Second, nonliving, solid substrates frequently exhibit distinct patterns of seasonal variation in moisture, whereas short-term fluctuations are dampened. Thus, these environments may provide insects with discernible seasonal cues. Moreover, experimentation with nonliving media is not confounded by the active uptake of water that characterizes living plant material. An additional advantage of nonliving solid material is that insects that inhabit these substrates often can be moved with little disturbance (e.g., within earthen cells or blocks of wood).

Crucial Issues. In addition to choosing the appropriate species, 3 crucial issues impinge upon or have hampered investigations of moisture as a seasonal cue. As illustrated below, each of these should be considered in the design of experiments dealing with moisture as a seasonal cue.

First, and perhaps most difficult, is to isolate the effects of moisture on specific phases of the life cycle (see Tauber et al. 1986, pp. 38-66). Because the physiological state and responses of insects change continually, accomplishing this task requires field-based knowledge of the seasonal cycles of the experimental animals, relative to seasonal changes in moisture and other physical factors. For example, typically, dryseason dormancy begins at the end of the rainy season and persists at least until conditions become very dry; subsequently, postdiapause activity is synchronized with the onset of the rainy season. Using the above information it is possible to construct 3 biologically meaningful hypotheses regarding moisture's role as a seasonal cue. First, because moist conditions prevail during prediapause periods of growth and development and during the early phases of dormancy, it is reasonable to hypothesize that moist (versus dry) conditions have a role in inducing or maintaining diapause. Second, because postdiapause development occurs during the dry season, we could appropriately hypothesize that dry or drying conditions have a role in terminating diapause. Finally, because reactivation or adult emergence consistently coincides with the onset of the rainy season, we would hypothesize that rainfall stimulates specific behavioral functions, such as emergence from the soil. Insects whose life cycles are correlated with moisture in a different manner would require other hypotheses, but in all cases, the development of specific, testable hypotheses requires knowledge of the timing of the insect life cycle relative to seasonal changes in major physical factors.

The 2nd challenge is to establish how insects perceive and respond to moisture in their environment. Insects may measure the absolute level of a seasonal stimulus or a change in the level of a stimulus (Beck 1980, Tauber et al. 1986, Danks 1987). And, they may 


\section{Month of transfer from field conditions}
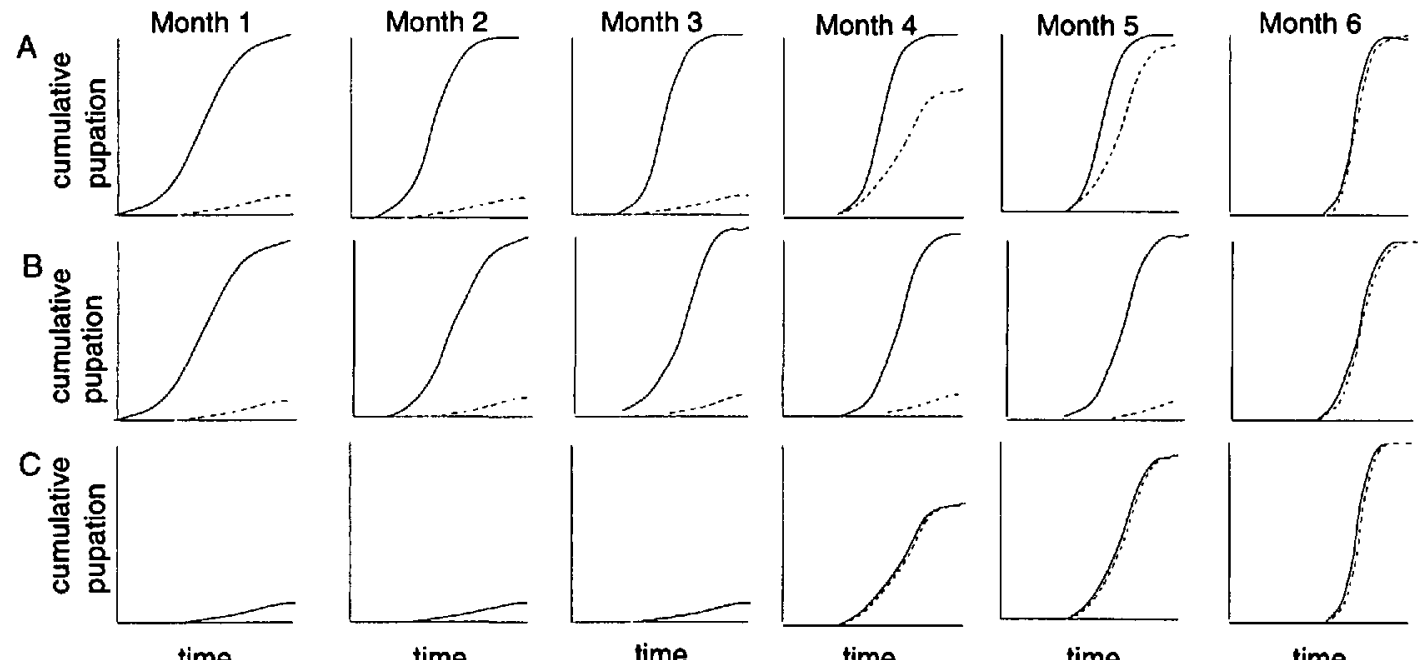

time

time

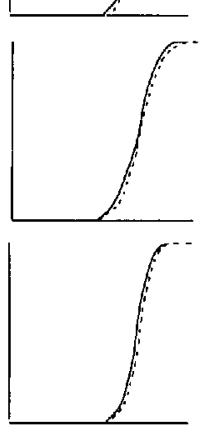

time

Fig. 1. Three possible results from experiment l: temporal patterns of diapause termination (=pupation) in larvae exposed to moist (dashed line) and dry (solid line) conditions. Figures from left to right represent the responses of larvae to 2 conditions in monthly tests throughout diapause. These seasonal patterns of response to moisture would lead us to the following conclusions: (A) Moist conditions maintain diapause; the gradual increase in the incidence of pupation suggests that diapause ends spontaneously, without a specific stimulus. (B) Moist conditions maintain diapause. An abrupt increase in pupation before the end of the dry season would lead us to suggest that a stimulus other than moisture, such as a cold period (see Janzen 1987), hastens diapause termination. An abrupt increase in pupation after the beginning of the rainy season indicates that moisture either terminates diapause or triggers pupation. (C) Similar patterns of pupation in dry and moist conditions indicate that moisture has no role in diapause maintenance or termination. If the pattern of pupation in the dry experimental treatments differs from the "unmoved" controls, then the tests would be inconclusive because disturbance influenced diapause. Any response to moisture could be quantified in subsequent tests.

react to these 2 types of seasonal cues with either an all-or-none (threshold) response or a quantitative (graded) response. Thus, experiments should be designed to establish the quantitative relationship between moisture and seasonal responses and to elucidate the mechanisms used in perception and response.

The 3rd issue in experimentally evaluating moisture's role as a seasonal cue centers on the sometimes formidable technical hurdles associated with moisture as a seasonal variable. For example, it is necessary to measure and monitor moisture levels and to standardize and manipulate moisture for relatively long periods (e.g., the full duration of the experiments) in a variety of media. Significantly, techniques now exist for accomplishing these objectives in soil and other environments that have presented formidable difficulties in the past (Crossley et al. 1991). For example, when soils (or other solid media, such as wood) are unsaturated, capillarity allows the slow redistribution of water throughout. However, relatively stable moisture stratification can be maintained if the width of each stratum is large and the differences in moisture levels between strata are small (Villani and Wright 1988, Villani and Nyrop 1991).

Because water moves relatively slowly and unevenly through unsaturated soil (and other media), it is often not feasible to change levels of soil moisture during experimentation, and therefore the animals must be moved among experimental chambers with different levels of moisture. With each transfer, 2 controls are necessary to differentiate between the effects of moisture and disturbance: 1 group must be moved to new arenas with the original levels of moisture, and a 2nd group remains in situ in the original moisture conditions.

\section{Experimental Paradigms}

Given the above considerations, we describe 3 experimental paradigms that delineate the effects of moisture on critical aspects of insect seasonal cycles (diapause, development, and seasonal behavior). To illustrate the main issues, we simplified the experimental conditions (usually wet versus dry soil). However, depending on the experimental goal, each experiment could be expanded to quantify the insects' responses to moisture and to identify how the insects perceive moisture as a seasonal cue. Although we tailored the experimental conditions to soil-inhabiting insects that undergo the dry season in a state of larval diapause, our approach offers a general experimental framework that can be adjusted to other life cycles, seasonal patterns of rainfall, or media by modifying the hypotheses and experimental conditions appropriately.

Experiment 1: Influence of Moisture on Diapause. The lst hypothesis (token stimulus hypothesis) postulates that moisture acts as a token stimulus (antic- 
ipatory cue) that influences diapause. Testing this hypothesis entails exposing insects to a range of moisture conditions at regular intervals, and measuring the effect on diapause induction, maintenance, or termination.

As an example, we focus on the role of moisture in the maintenance and termination of larval diapause that is initiated in mature larvae at the end of the rainy season and that extends into the dry season. Because of the timing of dormancy in the field, we expect that moist conditions maintain larval diapause (delay the termination of diapause) and that dry conditions have an active role in terminating diapause (signalled by pupation).

To test these ideas, diapausing larvae would be maintained in constantly moist media that simulate natural conditions at the beginning of diapause; whereas temperature would be adjusted to mimic the natural pattern of seasonal change. At regular (e.g., monthly) intervals during diapause, larvae would be moved to dry conditions and then held until diapause terminates. Appropriate controls would test for an effect of disturbance, as described above. During each monthly test, the temperature would be held constant at the level prevailing at the beginning of the month. Figure 1 shows the anticipated results.

Experiment 2: Influence of Moisture on Thermal Accumulation. The 2 nd hypothesis (developmental modulator hypothesis) states that moisture directly affects rates of growth, maturation, reproduction, or activity. Tests of this hypothesis entail measuring these parameters when animals are held under a single temperature regimen but a range of moisture levels.

In the experimental design, care must be taken to ensure that any effects of moisture on diapause or behavior (emergence, moulting) are excluded. Thus, our paradigm illustrates how to assess the impact of moisture on postdiapause development leading to pupal-adult ecdysis, without including the effect of moisture on either the termination of diapause or the imaginal moult. This is achieved in an experiment wherein both diapause termination (pupation) and ecdysis to the adult stage occur under similar conditions of moisture in all treatments (Fig. 2).

At the beginning of this experiment, larvae are allowed to terminate diapause (pupate) under simulated dry-season conditions. Immediately following the larval-pupal molt, individual pupae are allocated among 4 treatments that subject the animals to an equal period in moist soil, but at different times during the pupal stage (Fig. 2, top). Three additional groups serve as controls for effects of disturbance; they would be moved to and from dry, rather than moist soil, at the various times during the pupal stage

The lower half of Fig. 2 shows the anticipated results. In this example, temperature conditions would be identical in all treatments and would approximate the temperature that the insects would encounter naturally. The experiment could be expanded to identify an interaction between moisture and temperature, by conducting the same test under a broad range of temperatures and moisture levels.

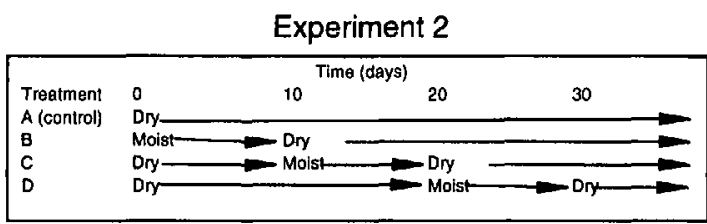

Pattern of response

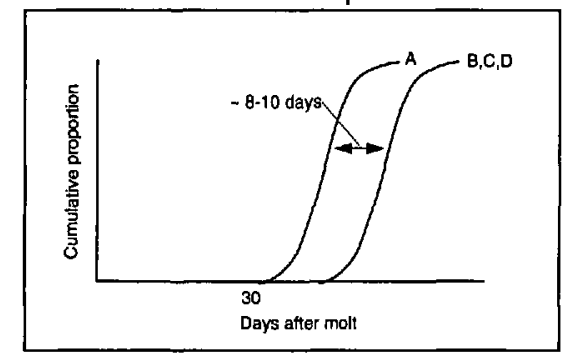

Fig. 2. Design and expected results for experiment 2. The experiment consists of 4 treatments in which postdiapausing insects are either kept continually dry (treatment $A$, control), or exposed at various times to moist conditions (treatments B-D). Cumulative proportion undergoing ecdysis in the control condition (treatment A) is expected to follow a logistic curve. If heat accumulation is curtailed by the experimental treatments, the emergence curves will be shifted to the right as shown for treatments $B, C$, and D. Each of treatments $B, C$, and D need not have the same effect, and some response profiles may be identical to $A$, indicating no effect of treatment. Differences in the timing of ecdysis between the group that remained in situ under dry conditions (treatment $\mathbf{A}$ ) and those moved to and from dry soil at various times during the pupal stage (3 additional controls, not figured) would indicate an effect of disturbance.

Experiment 3: Moisture as a Behavioral Stimulus. The 3rd hypothesis (behavioral cue hypothesis) postulates that moisture acts as a stimulus for vital seasonal events, such as postdiapause hatching, moulting, mating, or movement. To test this hypothesis, experiments would expose animals to a range of moisture conditions and to relative changes in moisture because the organisms may respond to either cue. Conceptually, this type of experiment is simple, but its execution may be formidable. Important considerations are how to: measure the particular behavior, maintain moisture levels precisely, alter relative moisture levels, and avoid introduction of confounding stimuli, such as physical movement.

For example, given a species in which adults begin their seasonal emergence soon after the initiation of heavy rains, an experiment that tests whether moisture stimulates postdiapause movement from the soil could be approached as follows. At the beginning of the experiment, postdiapause (preemergent) insects would be held in dry soil (or other solid medium) that simulates natural conditions before the rains begin. After a lapse of time (e.g., after the rainy season starts and emergence begins in the field), individual experimental animals would be transferred to arenas that differ in moisture, including one with no change, as a control (Fig. 3). An additional group would remain in situ as a 2 nd control for an effect of disturbance. Then, 

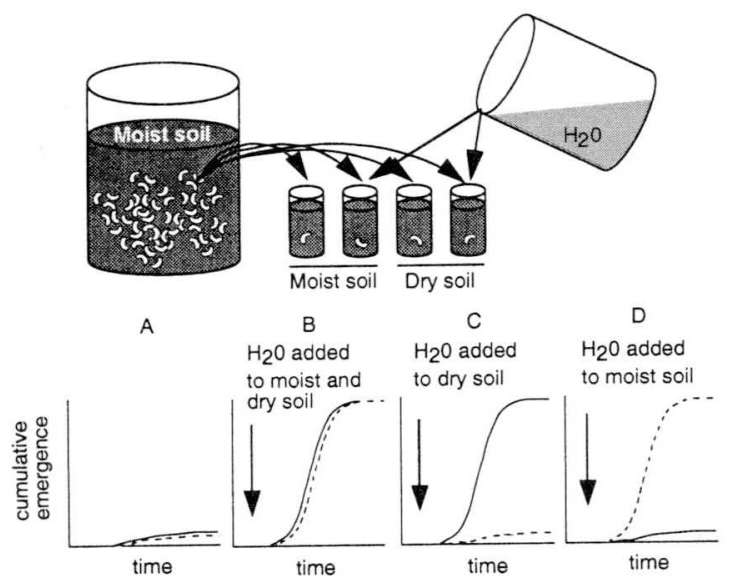

Fig. 3. Expected results for experiment 3 . If transferring preemergent insects to dry (solid line) and moist (dashed line) conditions result in similar, low levels of emergence (e.g., A), then the insects probably do not respond to absolute moisture levels; however, experiments with other levels of moisture should then be conducted to confirm this. Similar but high levels of emergence after the transfer would lead us to suspect an experimental artifact stemming from disturbance, and we would compare the emergence with that in the unmoved control. If passage of the water front in the dry and moist soils produces higher rates of emergence than the moist soil alone (cf., A, B), then it is reasonable to conclude that the insects respond to rainfall (contact water or ingestion). If there is a greater response to the descending water front among animals in dry soil than those in moist soil (C), then it appears that the insects respond to a change in moisture. If the combination of moist soil and the passage of a water front produces the highest levels of emergence (D), it is likely that emergence is influenced by an interaction between absolute and relative soil moisture.

to mimic the passage of rainfall through the medium, half of the arenas within each of the experimental conditions would be moistened with water having the same temperature as the medium. Movement of the adults through the medium could then be observed either directly or with radiography, and their emergence could be recorded.

If movement or emergence in any of the above treatments is greater than under dry conditions, it is reasonable to conclude that moisture influences behavior. Fig. 3 depicts the expected results.

Experiments can also be conducted to determine whether drying of the medium reverses or inhibits emergence. These tests would entail transferring preemergent insects to moist conditions and then drying the medium in half of the arenas.

In conclusion, a prime intellectual and practical challenge for basic and applied biologists is to clarify moisture's proximate and ultimate roles in the seasonal ecology of invertebrates. In offering our paradigm, we focus on the underlying ecophysiological aspects and propose that an elucidation of insect responses to moisture, especially in insects that inhabit soil or other nonliving solid media, represents one of the last frontiers in the quantitative analysis of insect life histories.
We hope to stimulate (1) investigations on the seasonal ecology of insects in predominantly wet-dry environments, (2) the development of practical, comprehensive insect phenological models that include moisture as a variable where appropriate, and (3) comparative evolutionary analyses of insect adaptations to wet-dry seasons. Our aim is to combine insights derived from natural-history studies with rigorous and technically feasible experiments on moisture's role as a seasonal factor. At the core of the approach is a series of hypotheses that are generally applicable to insects, other invertebrates, plants, and microbes and that draw parallels from the rigorously studied invertebrate responses to photoperiod and temperature. Data gathered from such experiments will lead to a general understanding of insect adaptations to seasonal environments and to improved ecologically based pest management systems, especially in regions with marked wet-dry seasons.

\section{Acknowledgments}

We thank A. M. Shapiro (University of California, Davis) and J. S. Clegg (Bodega Marine Laboratory, University of California, Davis) for their thoughtful comments on an earlier draft of the manuscript and J. L. Krysan (USDA-ARS) for his help during a critical period of our research (MJT, CAT). This work was supported, in part, by Regional Project 185 and Hatch Project 408.

\section{References Cited}

Ando, Y. 1972. Egg diapause and water absorption in the false melon beetle, Atrachya menetriesi Faldermann ( $\mathrm{Co}-$ leoptera: Chrysomelidae). Appl. Entomol. Zool. 7: 142154.

Beck, S. D. 1980. Insect photoperiodism, 2nd ed. Academic Press, New York.

Becker, G. 1977. Ecology and physiology of wood destroying Coleoptera in structural timber. Mater. Org. Berl. 12: 141-160.

Bonato, O., S. Mapangou-Divassa, and J. Gutierrez. 1995. Influence of relative humidity on life-history parameters of Mononychellus progresivus and Oligonychus gossypii (Acari: Tetranychidae). Environ. Entomol. 24: 841-845.

Bradshaw, W. E., and C. M. Holzapfel. 1988. Drought and the organization of tree-hole mosquito communities. Oecologia (Berl.) 74: 507-514.

Brakefield, P. M. 1987. Tropical dry and wet season polyphenism in the butterfly Melanitis leda (Satyrinae): phenotypic plasticity and climatic correlates. Biol. J. Linn. Soc. 31: 175-19.

Brakefield, P. M., and N. Reitsma. 1991. Phenotypic plasticity, seasonal climate and the population biology of Bicyclus butterflies (Satyridae) in Malawi. Ecol. Entomol. 16: 291-303.

Cammell, M. E., and J. D. Knight. 1992. Effects of climatic change on the population dynamics of crop pests. Adv. Ecol. Res. 22: 117-125.

Chu, P.-C., K. J. Giroux, and R. E. Stinner. 1992a. A dynamic model of water gain and loss in larval Mexican bean beetle. Physiol. Entomol. 17: 309-315.

Chu, P.-C., R. E.Stinner, and K. J. Giroux, 1992b. A dynamic model of larval Mexican bean beetle survival as a function of body water balance. Physiol. Entomol. 17: 316-324. 
Cloudsley-Thompson, J. L. 1991. Ecophysiology of desert arthropods and reptiles. Springer, Berlin, Germany.

Crossley, D. A., Jr., D. C. Coleman, P. F. Hendrix, W. Cheng, D. H. Wright, M. H. Beare, and C. A. Edwards [eds.]. 1991. Modern techniques in soil ecology. Elsevier, New York.

Danks, H. V. 1987. Insect dormancy: an ecological perspective. Biological Survey of Canada Monograph Series No. 1. Biological Survey of Canada (Terrestrial Arthropods), Ottawa, Canada.

Denlinger, D. L. 1986. Dormancy in tropical insects. Annu. Rev. Entomol. 31: 239-264.

Dobkin, D. S., I. Olivieri, and P. R. Ehrlich. 1987. Rainfall and the interaction of microclimate with larval resources in the population dynamics of checkerspot butterflies (Euphydryas editha) inhabiting serpentine grassland. Oecologia (Berl.) 71: 161-166.

Edney, E. B. 1977. Water balance in land arthropods. Zoophysiology and ecology, vol. 9. Springer, New York.

Essig, E. O. 1926. Insects of western North America. MacMillan, New York.

Fletcher, B. S., S. Pappas, and E. Kapatox. 1978. Changes in the ovaries of olive flies (Dacus oleae (Gmelin)) during the summer, and their relationship to temperature, humidity and fruit availability. Ecol. Entomol, 3: 99-107.

Gebre-Amlak, A. 1989. Termination of diapause in the maize stalk borer, Busseola fusca (Fuller) (Lep., Noctuidae), in Awassa, southern Ethiopia. J. Appl. Entomol. 107: 160-165.

Gehrken, U. 1989. Diapause termination in eggs of the stonefly Arcynopteryx compacta (McLachland) in relation to dehydration and cold hardiness. J. Insect Physiol. 35: $377-385$

Gutierrez, A. P., M. A. Pizzamiglio, W. J. Dos Santos, A. Villacorta, and K. D. Gallagher. 1986. Analysis of diapause induction and termination in Pectinophora gossypiella in Brazil. Environ. Entomol. 15; 494-500.

Hadley, N. F. 1994. Water relations of terrestrial arthropods. Academic Press, New York.

Ingrisch, S. 1986. The plurennial life cycles of the European Tettigoniidae (Insecta: Orthoptera). Oecologia (Berl.) 70: 624-630.

Janzen, D. H. [ed.]. 1983. Costa Rican natural history. University of Chicago Press, Chicago.

Janzen, D. H. 1987. How moths pass the dry season in a Costa Rican forest. Insect Sci. Appl. 8: 489-500.

Jones, R. E. 1987. Reproductive strategies for the seasonal tropics. Insect Sci. Appl. 8: 515-521.

Jordan, S., and S. F. Hubbard. 1991. Influence of humidity and temperature on the diel periodicity of oviposition of Toxorhynchites moctezuma (Diptera: Culicidae) in the field. J. Med. Entomol. 28: 241-245.

Kfir, R. 1993. Diapause termination in the spotted stem borer, Chilo partellus (Lepidoptera: Pyralidae) in the laboratory. Ann. Appl. Biol. 123: 1-7.

King, A.B.S. 1984. Biology and identification of white grubs (Phyllophaga) of economic importance in Central America. Trop. Pest Manage. 30: 36-50.

Labeyrie, V. [ed.]. 1981. The ecology of bruchids attacking legumes (Pulses). W. Junk, The Hague, The Netherlands.

Lee, R. E., Jr., and D. L. Denlinger. 1991. Insects at low temperature. Chapman \& Hall, New York.

Masaki, S. 1980. Summer diapause. Annu. Rev. Entomol. 25: 1-25.

Mattson, W. J., and R. A. Haack. 1987. The role of drought in outbreaks of plant-eating insects. Bioscience 37: 110118.
McLeod, L. 1984. Seasonal polyphenism in African Precis butterflies, pp. 313-315. In R. I. Vane-Wright and P. R. Ackery [eds.], The biology of butterflies. Symposium of the Royal Entomological Society of London, No. 11. Academic Press, London.

Mooney, H. A., P. R. Ehrlich, D. E. Lincoln, and K. S. Williams. 1980. Environmental controls on the seasonality of a drought deciduous shrub, Diplacus aurantiacus and its predator, the checkerspot butterfly, Euphydryas chalcedona. Oecologia (Berl.) 45: 143-146.

Okuda, T. 1990. Significance of water contact as a factor terminating larval diapause in a stem borer, Busseola fusca. Entomol. Exp. Appl, 57: 151-155.

1991. Enhancement of diapause development in a stem borer, Busseola fusca (Lep.: Noctuidae) by dry conditions. Appl. Entomol. Zool. 26: 49-5.

Paarmann, W. 1986. Seasonality and its control by environmental factors in tropical ground beetles (Col., Carabidae), pp. 157-171. In P. J. den Boer, M. L. Luff, D. Mossakowski, and F. Weber [eds.], Adaptations, dynamics and evolution of carabid beetles. Gustav Fischer, Stuttgart, Germany.

Parkin, E. A. 1943. The moisture content of timber in relittion to attack by Lyctus powder-post beetles. Ann. Appl. Biol. 30: 136-142.

Powell, J. A. 1986. Records of prolonged diapause in Lepidoptera. J. Res. Lepid. 25: 83-109.

Punyua, D. K., R. M. Newsib, and M. J. Mutinga. 1985. Diurnal and seasonal activity of unfed adult Rhipicephalis appendiculatus (Acarina: Ixodidae) in relation to some intrinsic and extrinsic factors- I Factors regulating activity. Insect Sci. Appl. 6: 63-70.

Régnière, J., R. L. Rabb, and R. E. Stinner, 1979. Popillia japonica: Effect of soil moisture and texture on survival and development of eggs and first instar grubs. Environ. Entomol. 10: 654-660.

Saunders, D. S. 1982. Insect clocks, 2nd ed. Pergamon, Oxford, England.

Shapiro, A. M. 1979. The phenology of Pieris napi microstriata (Lepidoptera: Pieridae) during and after the 1975-77 drought, and its evolutionary significance. Psyche (Camb.) 86: 1-10.

Sømme, L. 1995. Invertebrates in hot and cold arid environments. Springer, Berlin, Germany.

Spiller, D. A., and T. W. Schoener. 1995. Long-term variation in the effect of lizards on spider density is linked to rainfall. Oecologia (Berl.) 103: 133-139.

Tanaka, S. 1987. Changing responses to temperature and moisture of diapausing and developing eggs of Allonemobius fasciatus (Orthoptera: Gryllidae). J. Insect Physiol. 33: 635-641.

Tanaka, S., D. L. Denlinger, and H. Wolda. 1987a. Daylength and humidity as environmental cues for diapause termination in a tropical beetle. Physiol. Entomol. 12: 213-224.

Tanaka, S., H. Wolda, and D. L. Denlinger. 1987b. Seasonality and its physiological regulation in three neotropical insect taxa from Barro Colorado Island, Panama. Insect Sci. Applic. 8: 507-514.

Tauber, C. A., and M. J. Tauber. 1992. Phenotypic plasticity in Chrysoperla: genetic variation in the sensory mechanism and in correlated reproductive traits. Evolution 46 : 1754-1773

Tauber, M. J., C. A. Tauber, and S. Masaki. 1986. Seasonal adapatations of insects. Oxford University Press, New York,

Tauber, M. J., C. A. Tauber, and J. P. Nyrop. 1994. Soil moisture and post-dormancy emergence of Colorado po- 
tato beetles: a descriptive model and field emergence patterns. Environ. Entomol. 23: 1485-1496.

Taylor, F. 1989. Diapause induction in changing photoperiods. J. Theor. Biol. 139: 103-116.

van Houten, Y. M., and R. L. Veenendaal. 1990. Effects of photoperiod, temperature, food and relative humidity on the induction of diapause in the predatory mite Amblyseius potentillae. Exp. Appl. Acarol. 10: 111-128.

Villani, M. G., and J. P. Nyrop. 1991. Age dependent movement patterns of Japanese beetle and European chafer grubs in soil-turfgrass microcosms. Environ. Entomol. 20: 241-251.

Villani, M. G., and R. J. Wright. 1988. Use of radiography in behavioral studies of turfgrass-infesting scarab grub species (Coleoptera: Scarabaeidae). Bull. Entomol. Soc. Am. 34: 132-144.

Wagner, T. L., R. L. Olson, and J. L. Willers. 1991. Modeling arthropod development time. J. Agric. Entomol, 8: 25l270.

Wardhaugh, K. G. 1980. The effects of temperature and moisture on the inception of diapause in eggs of the
Australian plague locust, Chortoicetes terminifera Walker (Orthoptera: Acrididae). Aust. J. Ecol. 5: 187-191.

Williams, L. H. 1983. Wood moisture levels affect Xyletinus peltatus infestations. Environ. Entomol. 12: 135-140.

Wilson, K. G., R. E. Stinner, and R. L. Rabb. 1982. Effects of temperature, relative humidity, and host plant on larval survival of the Mexican bean beetle, Epilachna varivestis Mulsant. Environ. Entomol, 11: 121-126.

Wolda, H. 1978. Seasonal fluctuations in rainfall, food and abundance of tropical insects. J. Anim. Ecol. 47: 369-381. 1989. Seasonal cues in tropical organisms. Rainfall? Not necessarily! Oecologia (Berl.) 80: 437-442.

Wolda, H., and D. L. Denlinger. 1984. Diapause in a large aggregation of a tropical beetle. Ecol. Entomol. 9: 217 230.

Yoder, J. A., and D. L. Denlinger. 1992. Water vapour uptake by diapausing eggs of a tropical walking stick. Physiol. Entomol. 17: 97-103.

Received for publication 16 October 1997; accepted 5 January 1998. 